\title{
Studiengang und Fortbildung der Geographielehrer in Basel
}

Gesellschaft, Fach- und Bildungswissenschaften sind heute einem starken Wandel unterworfen. Die Lehrerausbildung muß auf diese Veränderungen reagieren.

Dieser kurze Beitrag soll - für das Schulfach Geographie und den Raum Basel - den heutigen Zustand in Aus- und Fortbildung darstellen und mögliche Entwicklungen aufzeigen.

\section{Der Ausbildungsgang im Überblick}

In Basel unterscheidet man zwischen Mittellehrern (ML, Sekundarstufe 1) und Oberlehrern (OL, Sekundarstufe 2). Die fachwissenschaftliche Ausbildung in drei Schulfächern dauert mindestens $6 \mathrm{Se}$ mester für ML bzw. 9 Semester für OL. Unter bestimmten Voraussetzungen wird das Lizenziat (oder Diplom) als wissenschaftlicher Oberlehrerabschluß anerkannt.

Die lehrerspezifische Ausbildung ist zweigeteilt: Fachdidaktik während des Studiums an der Universität und (nach dem wissenschaftlichen Abschluß) Fachmethodik und Erziehungswissenschaften am Kantonalen Lehrerseminar (Jahreskurs).

\section{Fachdidaktik an der Universität}

Das Geographische Institut hat sich im Wintersemester 1976/77, als erstes Institut der Universität Basel, an der Lehrerausbildung beteiligt (Abteilung Prof. Gallusser). Damals wurden «fachdidaktische Übungen» angeboten. Heute belegen die Studenten die Grundvorlesung Fachdidaktik 1 (2 Semesterstunden) mit den Schwerpunkten: Grundbegriffe der geographischen Fachdidaktik, Lernumfeld Schule, Lehrpläne, Lernziele und lernzielorientierter Unterricht, Unterrichtsplanung und Ordnen der Lerninhalte (Geschichte der Fachdidaktik Gg). Da für Bezirkslehrerkandidaten der Kantone Solothurn und Aargau nach dieser Vorlesung die fachdidaktische Ausbildung abgeschlossen ist, müssen auch die wichtigsten fachmethodischen Fragen behandelt werden. Übungen zur Vorlesung dienen ebenfalls diesem Zwecke: Schulbuchanalyse, Ope- rationalisieren von Lernzielen, Sachanalyse, Medienliste, Konzeption einer Unterrichtseinheit. Während den Veranstaltungen werden unterschiedliche Aktions- und Sozialformen des Schulunterrichts erprobt.

Seit dem Sommersemester 1977 ist im Ausbildungsprogramm Exkursionsdidaktik (1 Semesterstunde) enthalten. Nach einer Einführung werden die Grundlagen der Regionalgeographie erarbeitet. Danach stellen die Studenten selbständig Exkursionsprogramme zusammen. Begleitend führt der Dozent vier Stadt- und Landschaftsexkursionen durch.

Im Sommersemester 1989 wird neu Fachdidaktik 2 angeboten (2stündig, speziell für Oberlehrer). Ihre Zielsetzung kann wie folgt begründet werden:

Der Geographieunterricht an den Mittelschulen muß sich in Zukunft noch vermehrt den Problemen der gesellschaftlichen Wirklichkeit stellen. Zu den Lehrplanthemen wie Probleme der Entwicklungsländer, Ökologie und Raumplanung existieren speziell für die Basler Schulregion kaum aufgearbeitete Unterrichtsmaterialien. Geographielehrer sollten deshalb fähig sein, verschiedenste Quellen didaktisch zu sichten und für den eigenen Unterricht nutzbar zu machen. Wie kaum ein Fach bietet die Geographie die Möglichkeit, neue Forschungsergebnisse (oder Teile davon) für den Schulunterricht umzusetzen. Dies hat (nach ROHLEDER, 1987) sachgerecht (Vereinfachung zulässig), methodisch (angepaßte Unterrichtsmittel und -formen), sowie didaktisch (lernzielorientiert, stufenangepaßt) zu erfolgen. So muß denn die Schulgeographie «um erneuerungsfähig zu bleiben, in den direkten Kontakt mit der Forschung, d. h. mit den modernen Ansätzen der Geographie, treten. Dieser Kontakt soll auch den Abkoppelungstendenzen zwischen Hochschulund Mittelschul- bzw. Sekundarschulgeographie entgegenwirken» (WANNER, 1984, S. 159). Fachdidaktik 2 und die Fortbildungsveranstaltungen zielen in diese Richtung.

Peter Lüscher, Dr., Gymnasiallehrer, Lektor für Fachdidaktik Geographie, Geographisches Institut der Universität Basel 


\section{Fachmethodik am Kantonalen Lehrerseminar}

Nebst allgemeiner Didaktik, Pädagogik und Psychologie liegt das Schwergewicht der einjährigen Abschlußausbildung auf Fachmethodik und Unterrichtspraxis. Während zwei Wochenstunden werden die Kandidaten mit den methodischen Problemen des Geographieunterrichts (Stoffpläne, Präparation, Einstieg, Motivation, Medien u. a.) vertraut gemacht. Sie können dies in ihren eigenen Klassen (ganzjährige Vikariate bis zu 8 Wochenstunden) oder während eines 16stündigen Unterrichtspraktikums anwenden.

\section{Fortbildung der Geographielehrer}

Entscheidende Impulse für die Schulgeographie der Region Basel gingen vom 18. Deutschen Schulgeographentag aus (LÜSCHER, 1982), welcher in Basel durchgeführt wurde (Prof. H. Leser Vorsitzender des Organisationskomitees). So begann eine wichtige Phase in der Lehrerfortbildung mit 3-4 Veranstaltungen - jeweils im Wintersemester - unter dem Oberthema einer der Grunddaseinsfunktionen. Das Sommersemester ist den Exkursionen in die Regio Basiliensis vorbehalten. Dabei können Geographielehrer aller Stufen auf die finanzielle und organisatorische Unterstützung des ULEF (Institut für Unterrichtsfragen und Lehrerfortbildung, Basel) zählen. Als Referenten stellen sich Dozenten und Assistenten des Geographischen Instituts oder Fachleute aus der Praxis zur Verfügung. Um die Kontakte Hochschule-Schule weiter ausbauen zu können, ist für das Wintersemester 1989/90 am Geographischen Institut ein «Kolloquium für Geographielehrer», unter Mithilfe der beiden genannten Institute, geplant. Es ist zu hoffen, daß auch die Studierenden an diesen 3-4 Vorabendveranstaltungen teilnehmen werden.

\section{Zukunftsprobleme - und Aufgaben der Basler Schulgeographie}

Für alle in der Lehrerausbildung Tätigen stellt die ungewisse Zukunft ihrer Studenten ein großes Pro- blem dar. Dies besonders auch im Fachbereich Geographie. Der Geburtenrückgang zu Beginn der 80er Jahre wird nach einer Studie des CESDOC (1988) an den Gymnasien im Einzugsgebiet der Universität (Kantone BS, BL, AG und SO) bis 1996 zu einem Verlust von etwa 9 vollen Geographiepensen führen. Nur etwa 5 davon können durch Pensionierung kompensiert werden. Fachwissenschaft und Fachdidaktik werden ihre Studenten in Zukunft sowohl auf die Lehrtätigkeit wie auch auf eine größere berufliche Mobilität hin ausbilden müssen.

Schulgeographen und Fachdidaktiker in Basel müssen in unmittelbarer Zukunft zu verschiedenen Problemen und Fragenkreisen Stellung beziehen. $\mathrm{Zu}$ nennen sind etwa:

- Ökologieunterricht (Beitrag der Geographie?)

- Informatikunterricht und Geographie?

- Interdisziplinärer Unterricht (Was kann die Geographie bieten? Was benötigt der Geographieunterricht von anderen Schulfächern?)

- An den Schulen unserer Region nimmt der Ausländeranteil stark zu (Auswirkungen auf den Geographieunterricht?)

- Legitimationsdruck auf das Fach bei Diskussionen um die Stundentafel einzelner Schulstufen.

- Geographie in der Erwachsenenbildung.

Der Ausbau der Fachdidaktischen Abteilung am Geographischen Institut, wie er hier skizziert und im «Leitbild Geographie Schweiz» gefordert wird (LeIMgRuber, 1988), ist ein wichtiger Schritt in die Zukunft der Basler Schulgeographie.

\section{Literatur}

CESDOC (1988): Prognosen der an den Maturitäts- und Diplommittelschulen zu erteilenden Lektionen, Schweiz. Dokumentationsstelle für Schul- und Bildungsfragen, Statistische Reihe Nr. 3, Genf (dazu eine Loseblättersammlung der Kantonsergebnisse $\mathrm{BS}, \mathrm{BL}, \mathrm{AG}, \mathrm{SO}$ )

LEIMGRUBER, W. (1988, Redaktion): Leitbild Geographie Schweiz. In: Geographica Helvetica Nr. 1, S. 33-36

LÜSCHER, P. (1982): Geographieunterricht im Wandel - ein Beitrag aus schweizerischer und baslerischer Sicht. In: Basler Schulblatt Nr. 12, Basel

ROHLEDER, M. (1987): Geographie hat Zukunft. In: Praxis Geographie Heft 7/8, S. 72-74, Braunschweig

WANNER, H. (1984): Schulgeographie und Pradigmenwechsel. In: Geographica Helvetica Nr. 3, S. 156-160 